JPE 11-3-11

\title{
Piecewise Affine Control Design for Power Factor Correction Rectifiers
}

\author{
Farzad Tahami ${ }^{\dagger}$, Shahab Poshtkouhi*, and Hamed Molla Ahmadian** \\ ${ }^{\dagger}$ Dept. of Electrical Eng., Sharif University of Technology, Tehran, Iran \\ * Dept. of Electrical and Computer Eng., University of Toronto, Toronto, Canada \\ ** Dept. of Electrical Eng., Ferdowsi University of Mashhad, Mashhad, Iran
}

\begin{abstract}
Single-phase power factor correction (PFC) converter circuits are non-linear systems due to the contribution of their multiplier. This non-linearity causes difficulties in analysis and design. Models that reduce the system to a linear system involve considerable approximation, and produce results that are susceptible to instability problems. In this paper a piecewise affine (PWA) system is introduced for describing the nonlinear averaged model. Then robust output feedback controllers are established in terms of the linear matrix inequality (LMI). Simulation and experiments results show the effectiveness of the proposed control method.
\end{abstract}

Key Words: Converter modeling, Linear matrix inequality (LMI), Piecewise affine (PWA) systems, PFC rectifiers

\section{INTRODUCTION}

Conventional diode peak-detector rectifiers draw a pulsating ac line current from the utility grid. As a result, they inject high order harmonic components to the utility line. Power factor correction (PFC) rectifiers offer several well-known benefits, including automatic line-voltage adjustments and power quality improvement. PFC rectifiers are now commonly used in low power supply systems connected to AC networks providing input-line harmonics in accordance with harmonic distortion standards. The control system allows the rectifier input current to track the input voltage, by emulating a loss free resistor. Thus, a close to unity power factor is achieved [1].

A variety of approaches are known for resistor emulation purposes. The most popular is a multiplying controller. A conventional PFC rectifier is based on a PWM converter controlled by two interconnected feedback loops, a wide bandwidth current loop and a slow voltage loop. To obtain a dynamic model of the rectifier we only need to model the dominant behavior of the system, i.e., how slow variations in the control signal, the load and the input affect the rectifier output. The use of averaging has been well accepted as a way to model the low-frequency components of the waveforms in a switching converter. The idea behind averaged circuit modeling is to identify a switch cell that is common in different topologies and to develop an equivalent circuit that,

\footnotetext{
Manuscript received Oct. 9, 2010; revised Mar. 15, 2011

Recommended for publication by Associate Editor Se-Kyo Chung.

$\dagger$ Corresponding Author: tahami@sharif.ir

Tel: +98-21-66165961, Fax: +98-21-66023261, Sharif University of Tech.

* Dept. of Electrical and Computer Eng., University of Toronto, Canada

** Dept. of Electrical Eng., Ferdowsi University of Mashhad, Iran
}

when inserted in place of the original switch cell, results in an electrical circuit that has the same average behavior as the converter [2].

Generally the averaged model of PWM converters is nonlinear. A small-signal model may be obtained by linearizing the large-signal averaged model by about one operating point. The main drawback of small-signal analysis is its inability to reveal system behavior under large-signal perturbations. Large signal transients can occur under various conditions such as source fluctuations, load switching and component failures. Actually, many systems, such as PFC rectifiers demand large signal modeling and analysis. The nonlinear time-varying nature of a system does not allow linearization of the equations needed to design rectifier averaged current controllers for most converter topologies [3]. There are few major shortcomings to linear analyses of PFC rectifiers. The averaged model of a multiloop dc-dc converter may have quadratic nonlinearity. Hence, such a system may have more than one equilibrium solution. If two of these solutions are stable, then the system will have two operating points, one of which is the nominal solution. This possibility is completely ignored in linearized averaged models. Consequently, the small-signal model cannot predict the post-instability dynamics. Besides, controllers that are designed based on this model may be conservative and may not achieve globally stable closed-loop systems. Therefore, it is essential to investigate the stability of PFC converters by using a non linear model.

Nonlinear stability analyses have been investigated for PWM converters, but they were limited to simple systems, such as single converter input filter systems, or systems with special structures [4]. Recently, nonlinear controls for single phase unity power factor rectifiers have been proposed by 


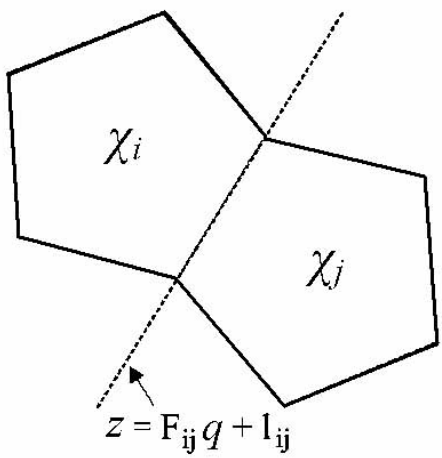

Fig. 1. Neighboring polytopic cells and their common boundary.

some researchers [5], [6]. Digital control methods have been studied for PFC rectifiers by other researchers [7], [8].

Over the last few years promising new methods have emerged for the analysis of piecewise-linear and piecewiseaffine systems. These systems represent a powerful model class for nonlinear systems. They arise naturally from linear dynamics in the presence of saturations or as simple examples of hybrid systems where the continuous dynamics within the different discrete states is linear or affine [9]. Piecewiseaffine (PWA) systems can also be used to approximate other nonlinear systems.

The authors have already extended the control methods designed for PWA systems to the nonlinear system arising from the averaging of PWM converters [10]. The authors have also developed a PWA modeling technique for PFC rectifier circuits [14]. In this paper this modeling is used to design piecewise linear (PWL) output feedback controllers for PFC rectifiers. The controllers are designed by solving an optimization problem with a set of linear matrix inequality (LMI) constraints. The local robust controllers ensure the stability of the rectifier against variations in system parameters and in the operating point. The effectiveness of this modeling and controller design method for PFC rectifiers is verified by simulations and experiments.

\section{PWA SYSTEM APPROXIMATION}

Many of the nonlinearities that frequently appear in engineering systems are either piecewise-affine or can be approximated as piecewise-affine functions. Piecewise-affine systems are a particular class of hybrid systems that offer a good modeling framework for complex dynamical systems involving nonlinear phenomena. PWA systems are defined by a series of affine systems over non-overlapping polytopic sets in the state-input space. A nonlinear system can be represented over a wide range of an operating region by combining the local polytopic models. The polytopic model defined herein has a structure that is particularly amenable to searching for Lyapunov function candidates. Consider a nonlinear system described by the following equations:

$$
\begin{aligned}
& \dot{z}(t)=f(z)+h(z) u \\
& y=C z
\end{aligned} .
$$

It is assumed that a partition of the state space with polytopic cells $R_{i}, i \in I$ is given. Each cell is constructed as the intersection of finite number of half spaces $\chi_{i}, i \in I$ [9]:

$$
\chi_{i}=\left\{z \mid H_{i}^{T} z-g_{i}<0\right\}
$$

where, $H_{i}=\left[h_{i 1} h_{i 2} \ldots h_{i p_{i}}\right]$, and $g_{i}=\left[g_{i 1} g_{i 2} \ldots g_{i p_{i}}\right]$ are constant vectors that define the partitioning.

Any two cells sharing a common facet will be called neighboring cells as shown in Fig. 1. A parametric description of the boundaries can then be obtained [9]:

$$
\chi_{i} \bigcap \chi_{j} \subseteq\left\{l_{i j}+F_{i j} q \mid q \in R^{n-1}\right\}
$$

For $i=1, \ldots, M$ and $j=1, \ldots, N_{i}$, where $M$ is the number of cells and $N_{i}$ is the number of cells neighboring cell $i$.

It is further assumed that within each cell the dynamics of the plant can be approximated by an affine system defined by:

$$
G_{i}\left\{\begin{array}{l}
\dot{z}(t)=A_{i} z(t)+b_{i}+B_{i} u(t) \\
y(t)=C z(t)
\end{array} .\right.
$$

Finding the piecewise-affine approximation of a nonlinear function based on its values at the vertices of a simplicial grid is basically an estimation problem. Given $f: R^{n} \rightarrow R^{n}$ and $n+1$ vertices belonging to a simplicial cell $i$, the objective is to find the matrix $A_{i}$ and the vector $b_{i}$ such that the piecewise-affine approximation of $f$ in the polytopic cell $i$ is described by:

$$
f(z) \approx A_{i} z+b_{i} .
$$

At each vertex of each simplex $i$, a linear equation of the form $f(\alpha)^{T}=\left[\alpha^{T} \mid 1\right] \theta$ can be written, where $\theta$ is defined by:

$$
\theta=\left[A_{i}^{T} \mid b_{i}^{T}\right]^{T} .
$$

And $\alpha$ represents the $n \times 1$ vector with the coordinates of the vertex. If all the values $f(\alpha)$ are stacked in a matrix $F$ and all the rows $\left[\alpha^{T} \mid 1\right]$ are in a matrix $X$, the solution is then given by $\theta=X^{-1} F$. Since a simplicial partition of the domain of $f$ is being used, the matrix $X$ is nonsingular [12].

At this point, the matrix $B_{i}$ should be found for each simplicial cell. This is done by introducing the Chebychev center $w_{i}^{(c h e b)}$ for each cell, that is the center of an Euclidean ball with the maximum radius that can be fit inside the polytopic cell, and then using the following approximation [12]:

$$
B_{i}=h\left(w_{i}^{(c h e b)}\right) .
$$

Once the PWA approximation of the system is constructed, linear control techniques can be utilized to synthesize appropriate controllers for each cell. The principle of operation for the overall control system is shown in Fig. 2.

The switching among plant models is governed by the state $\mathrm{z}(t)$ and the input $u(t)$. Stability of the original system is obtained if it is possible to find a Lyapunov function that is valid for the bounding systems in all regions [13]. 


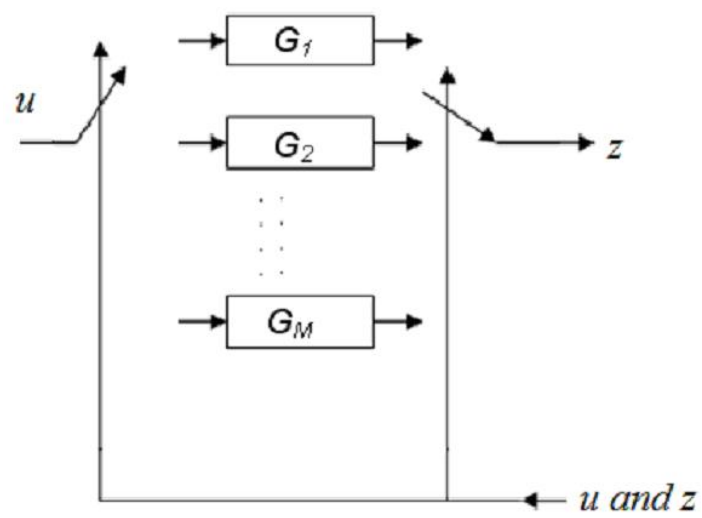

Fig. 2. The switching among PWA models.

\section{PWA MODEL DERIVATION AND CONTROLLER DESIGN}

\section{A. Average Signal Model of a Boost PFC Rectifier}

The simplest and less expensive approach to realizing a near-ideal rectifier is to employ a full-wave rectifier followed by a dc-dc converter. Among the different dc-dc converters, the boost converter is the most suitable for use in implementing PFC rectifiers. This is because the inductor is in series with the line input terminal, which achieves a smaller current ripple resulting in easier implementation of the average current mode control [14]. For illustrative purposes, a boost PFC rectifier is investigated in this paper. In the case of a boost converter the small signal assumption continues to be valid [1] allowing us to use simple linear controllers. However, this example is just to illustrate how the PWA controllers for PFC rectifiers can be constructed. Furthermore, by using this method the stability of the system can be guaranteed over the entire range of operation. A realized ideal rectifier using a boost PWM converter is depicted in Fig. 3. In general, near ideal rectifier systems contain two quite different types of control systems. The inner wide-bandwidth controller causes the instantaneous input current waveform to follow the input voltage by emulating a loss-free resistor. The outer control system maintains a power balance, stabilizing the rectifier output voltage against variations in load power, ac line voltage, and component values. Typically, a low harmonic distortion is achieved by limiting the bandwidth of the voltage loop to a fraction of the line frequency in order to properly attenuate the second line harmonic that appears at the output voltage of the converter. The assumption that the inner current loop operates ideally is usually sufficient to linearize the equations of the average voltage controller, but the nonlinear timevarying nature of the system does not allow linearization of the equations needed to design the rectifier averaged current controllers of most converter topologies [1].

To aid in the design of the inner feedback loop that controls the AC line current waveform, a converter model is needed that describes how the converter average input current depends on the duty cycle.

To illustrate this process, the large-signal model for a boost PFC rectifier, in which the MOSFET and diode conduction losses, as well as the inductor resistance are accounted for, is explained. It is assumed that the converter always operates in CCM. The averaged values of the inductor current $i_{g}$ and the energy storage capacitor voltage $v$ over one switching period are chosen as state variables. Employing the averaging technique, the state space representation of the circuit can be described as follows [14]:

$$
\dot{x}=A x+b(t)+g(X) u
$$

with:

$$
\begin{aligned}
& x=\left[\begin{array}{l}
x_{1} \\
x_{2}
\end{array}\right]=\left[\begin{array}{l}
i_{g} \\
v
\end{array}\right], A=\left[\begin{array}{cc}
-\frac{R_{L}}{L} & -\frac{1}{L} \\
\frac{1}{C} & -\frac{1}{R C}
\end{array}\right], b(t)=\left[\begin{array}{c}
\frac{v_{g}-V_{D}}{L} \\
0
\end{array}\right] \\
& g(x)=\left[\frac{\frac{V_{D}+x_{2}-R_{\text {on }} x_{1}}{L_{x_{1}}}}{-\frac{x_{1}}{C}}\right], u=d(t),
\end{aligned}
$$

where $R$ is the load resistance, $R_{o n}$ is the MOSFET ON resistance, $V_{D}$ is the diode forward voltage drop, and $R_{L}$ is resistance of the inductor.

\section{B. PWA Model Derivation}

The current loop model described by (8) represents a system which is nonlinear in input. Generally the small signal assumptions are violated, since the variations in the duty cycle, as well as in the ac input and current are not small [1].

To perform the piecewise-affine approximation it is assumed that a rectangular grid is provided for the domain of the nonlinearity. Given this grid, two basic steps remain. First, the piecewise-affine approximation of the nonlinearity within each cell of the partition is derived. Then, a polytopic description of the cells and a parametric description of the boundaries between the cells is created. Each cell of the partition will have a closed loop equilibrium at a point that must be specified.

To make the system of (8) be affine in $x$, a partitioning in the input voltage is also introduced. Following the method described in the last section, the dynamics of each cell can be described by following matrices:

$$
\begin{aligned}
A_{i} & =\left[\begin{array}{cc}
-\frac{R_{L}}{L} & -\frac{1}{L} \\
\frac{1}{C} & -\frac{1}{R C}
\end{array}\right] \\
b_{i} & =\left[\begin{array}{c}
\frac{v_{g, c h e b}-V_{D}}{L} \\
0
\end{array}\right] \\
B_{i} & =\left[\begin{array}{c}
\frac{V_{D}+x_{\text {cheb }}^{i 2}-R_{\text {on }} x_{\text {cheb }}^{i 1}}{L} \\
\frac{x_{\text {cheb }}^{i 1}}{C}
\end{array}\right]
\end{aligned}
$$

The polytopic regions are described as below:

$$
\begin{aligned}
\chi_{i} & =\left\{x \mid H_{i}^{T} x-g_{i}<0\right\}, \quad i=1,2, \ldots, M \\
x & =\left[\begin{array}{llllll}
x_{1} & x_{2} & u
\end{array}\right] \\
H_{i} & =\left[\begin{array}{cccccc}
-1 & 1 & 0 & 0 & 0 & 0 \\
0 & 0 & -1 & 1 & 0 & 0 \\
0 & 0 & 0 & 0 & -1 & 1
\end{array}\right] \\
g_{i} & =\left[-x_{1}^{-}\left(\chi_{i}\right) x_{1}^{+}\left(\chi_{i}\right)-x_{2}^{-}\left(\chi_{i}\right) x_{2}^{+}\left(\chi_{i}\right)-u^{-}\left(\chi_{i}\right) u^{+}\left(\chi_{i}\right)\right]^{T}
\end{aligned}
$$

where $x_{1}^{+}\left(\chi_{i}\right), x_{2}^{+}\left(\chi_{i}\right)$ and $u^{+}\left(\chi_{i}\right)$ represent the upper bounds and $x_{1}^{-}\left(\chi_{i}\right), x_{2}^{-}\left(\chi_{i}\right)$ and $u^{-}\left(\chi_{i}\right)$ represent the lower bounds of 


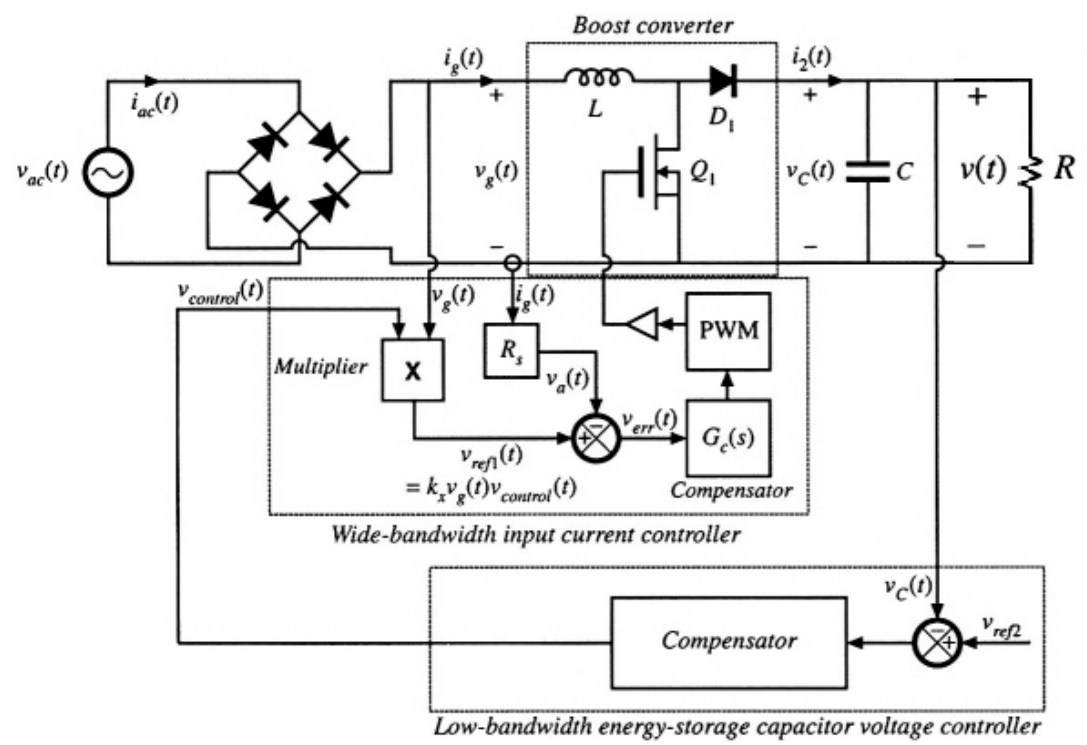

Fig. 3. Realizing of an ideal rectifier by a PWM converter [1].

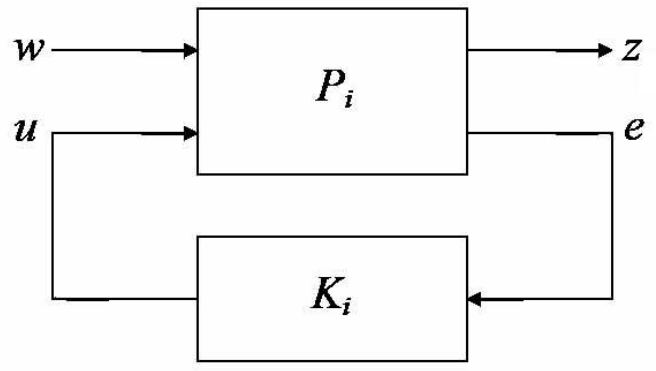

Fig. 4. Augmented plant and controller [14].

$x_{1}, x_{2}$ and $u$ in the cell $\chi_{i}$. Thus the linear feedback design methods can be applied.

\section{Controller Synthesis}

This section addresses a robust $H_{\infty}$ control problem for each cell in the sense of a dynamic output feedback. The controller minimizes the $H_{\infty}$ norm of the weighted tracking error in every region of the state space. The necessary and sufficient conditions for the existence of strong robust $H_{\infty}$ dynamic compensators and static output feedback controllers are established.

Considering the $i_{t h}$ cell of the state space, an affine model may be defined through the following equations:

$$
\begin{aligned}
& \dot{x}(t)=A_{i} x(t)+B_{i} u(t)+b_{i} \\
& y(t)=C x(t) .
\end{aligned}
$$

Using loop-shaping ideas and by introducing appropriate performance outputs and external inputs, an augmented PWL plant may be constructed as shown in Fig. 4 [15].

The state-space representation of the augmented plant $P_{i}$ may be described as follow:

$$
P_{i}\left\{\begin{array}{l}
\dot{x}(t)=A_{i} x(t)+B_{i}^{(1)} w(t)+B_{i}^{(2)} u(t) \\
z(t)=C_{i}^{(z)} x(t)+D_{i}^{(z 1)} w(t)+D_{i}^{(z 2)} u(t) \\
e(t)=C_{i}^{(e)} x(t)+D_{i}^{(e 1)} w(t)
\end{array}\right.
$$

where $w(t)$ is the reference input, and $e(t) \in R^{q}$ is the tracking error.

Now an output feedback controller for each cell is introduced. The state space representation of the controller is defined by:

$$
K_{i}\left\{\begin{array}{l}
\dot{\zeta}(t)=A_{i}^{(k)} \zeta(t)+B_{i}^{(k)} e(t) \\
u(t)=C_{i}^{(k)} \zeta(t)+D_{i}^{(k)} e(t)
\end{array}\right.
$$

where $\zeta \in R^{k}$ is the state variable vector of the controller. By applying the controller of (14) to the system of (13) the PWL closed loop system can be constructed as follows:

$$
\begin{aligned}
& \dot{x}_{c l}(t)=A_{i}^{(c l)} x_{c l}(t)+B_{i}^{(c l)} w(t) \\
& z(t)=C_{i}^{(c l)} x_{c l}(t)+D_{i}^{(c l)} w(t)
\end{aligned}
$$

where $x_{c l}=\left[\begin{array}{ll}x^{T} & \zeta^{T}\end{array}\right]^{T}$ is the closed loop state variable vector. The closed loop system matrices are obtained as follows:

$$
\begin{aligned}
A_{i}^{(c l)} & =\left[\begin{array}{cc}
A_{i}+B_{i}^{(2)} D_{i}^{(k)} C_{i}^{(e)} & B_{i}^{(2)} C_{i}^{(k)} \\
B_{i}^{(k)} C_{i}^{(e)} & A_{i}^{(k)}
\end{array}\right] \\
B_{i}^{(c l)} & =\left[\begin{array}{c}
B_{i}^{(1)}+B_{i}^{(2)} D_{i}^{(k)} D_{i}^{(e 1)} \\
B_{i}^{(k)} D_{i}^{(e 1)}
\end{array}\right] \\
C_{i}^{(c l)} & =\left[\begin{array}{ll}
C_{i}^{(z)}+D_{i}^{(z 2)} D_{i}^{(k)} C_{i}^{(e)} & D_{i}^{(z 2)} C_{i}^{(k)}
\end{array}\right] \\
D_{i}^{(c l)} & =D_{i}^{(z 1)}+D_{i}^{(z 2)} D_{i}^{(k)} D_{i}^{(e 1)} .
\end{aligned}
$$

Now the objective is to construct the transfer function of the $i_{t h}$ closed loop system from $w$ to $z$ and to minimize its $H_{\infty}$ performance subject to the disturbance attenuation factor $\gamma_{i}$. The $H_{\infty}$ control problem in linear systems can be cast into the form of first-order matrix polynomial inequalities called linear matrix inequalities (LMIs), which belong to the group of convex problems [16]. A system is said to be quadratically stable if there exists a positive-definite quadratic Lyapunov function that decreases along every trajectory of the system. A necessary and sufficient condition for quadratic stability can 
TABLE I

PARAMETERS OF PFC RECTIFIER

\begin{tabular}{|c|c|c|}
\hline Symbol & Parameter & value \\
\hline $\mathrm{C}$ & Filter Capacitor & $470 \mathrm{mu} \mathrm{F}$ \\
$\mathrm{L}_{\mathrm{f}}$ & Boost Inductor & $8 \mathrm{mH}$ \\
$\mathrm{R}_{\mathrm{ON}}$ & MOSFET on-resistance & $0.3 \Omega$ \\
$\mathrm{V}_{\mathrm{D}}$ & Diode forward voltage drop & $0.7 \mathrm{~V}$ \\
$\mathrm{R}_{\mathrm{L}}$ & Inductor resistance & $1.4 \Omega$ \\
$\mathrm{v}_{\mathrm{g}}$ & Input voltage (rms) & $110 \mathrm{~V}$ \\
$\mathrm{~V}$ & Output voltage & $200 \mathrm{~V}$ \\
$\mathrm{f}_{\mathrm{ac}}$ & Input frequency & $50 \mathrm{~Hz}$ \\
$\mathrm{f}_{\mathrm{s}}$ & Switching frequency & $40 \mathrm{kHz}$ \\
Pout & Output Power & $250 \mathrm{~W}$ \\
\hline
\end{tabular}

be directly formulated in terms of a finite number of linear matrix inequalities [17].

It can be shown that the LMI optimization problem is to minimize $\lambda^{2} \gamma_{i}-\alpha_{i}$ subject to the following constraints [10]:

$$
\begin{gathered}
{\left[\begin{array}{ll}
\varphi_{11} & \varphi_{12} \\
\varphi_{12}^{T} & \varphi_{22}
\end{array}\right]<0} \\
{\left[\begin{array}{cc}
R_{i} & \alpha_{i} I \\
\alpha_{i} I & S_{i}
\end{array}\right]>0} \\
\alpha_{i}-1>0
\end{gathered}
$$

where $\lambda^{2}$ is a weighting factor. The matrices $S_{i} \in R^{m \times m}$ and $R_{i} \in R^{m \times m}$ are LMI optimization variables and are defined through the following partitioning of $P_{i}$ [10]:

$$
P_{i}=\left[\begin{array}{cc}
S_{i} & N_{i} \\
N_{i}^{T} & U_{i}
\end{array}\right]
$$

subject to:

$$
P_{i}\left[\begin{array}{cc}
R_{i} & I \\
M_{i}^{T} & 0
\end{array}\right]=\left[\begin{array}{cc}
I & S_{i} \\
0 & N_{i}^{T}
\end{array}\right]
$$

where $N_{i} \in R^{m \times k}, M_{i} \in R^{m \times k}, U_{i} \in R^{k \times k}, m$ and $k$ are number of the system and controller states, respectively.

The parameters $\varphi_{11}, \varphi_{12}, \varphi_{22}$ are defined as follows:

$$
\varphi_{11}=
$$

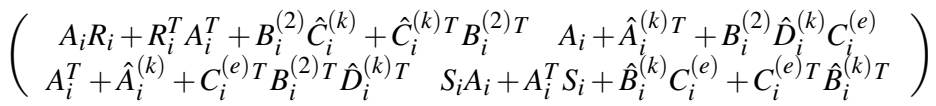

$$
\begin{aligned}
& \varphi_{12}=\left(\begin{array}{cc}
B_{i}^{(1)}+B_{i}^{(2)} \hat{D}_{i}^{(k)} D_{i}^{(e 1)} & R_{i} C_{i}^{(z)} T+\hat{C}_{i}^{(k)} T D_{i}^{(z 2)} \\
S_{i} B_{i}^{(1)}+\hat{B}_{i}^{(k)} D_{i}^{(e 1)} & C_{i}^{(Z)} T+C_{i}^{(e)} \hat{D}_{i}^{(k)} D_{i}^{(Z 2)} T
\end{array}\right) \\
& \varphi_{22}=\left(\begin{array}{cc}
-\gamma_{i} I & \left(D_{i}^{(z 1)}+D_{i}^{(z 2)} \hat{D}_{i}^{(k)} D_{i}^{(e 1)}\right)^{T} \\
D_{i}^{(z 1)}+D_{i}^{(z 2)} \hat{D}_{i}^{(k)} D_{i}^{(e 1)} & -\gamma_{i} I
\end{array}\right)
\end{aligned}
$$

This optimization problem can be solved using appropriate software. In the past decade, LMI solvers have emerged as powerful tools to solve the convex optimization problems that arise in many analysis and design applications. The MATLAB LMI Control Toolbox provides state-of-the-art optimization routines to solve LMIs. It also includes specialized tools for LMI based analysis and design of control systems [17]. Once the above LMI problem is solved, the nonsingular matrices $M$ and $N$ can be found. Then the controller parameters can be obtained [16].
TABLE II

CONTROLLERS DESIGNED FOR EACH CELL

\begin{tabular}{|c|c|}
\hline Cell & Controller \\
\hline 1 & $\frac{1.116 \mathrm{e} 0112 \mathrm{~s}+1.021 \mathrm{e} 016}{\mathrm{~s}^{2}+7.987 \mathrm{e} 009 \mathrm{~s}+2.501 \mathrm{e} 015}$ \\
3 & $\frac{7.483 \mathrm{e} 011 \mathrm{~s}+2.001 \mathrm{e} 016}{\mathrm{~s}^{2}+1.172 \mathrm{e} 010 \mathrm{~s}+4.515 \mathrm{e} 015}$ \\
4 & $\frac{3.448 \mathrm{e} 011 \mathrm{~s}+6.24 \mathrm{e} 015}{\mathrm{~s}^{2}+4.653 \mathrm{e} 009 \mathrm{~s}+1.892 \mathrm{e} 014}$ \\
5 & $\frac{3.295 \mathrm{e} 011 \mathrm{~s}+5.043 \mathrm{e} 015}{\mathrm{~s}^{2}+5.355 \mathrm{e} 09 \mathrm{~s}+9.686 \mathrm{e} 012}$ \\
6 & $\frac{2.838 \mathrm{e} 011 \mathrm{~s}+9.49 \mathrm{e} 015}{\mathrm{~s}^{2}+5.564 \mathrm{e} 09 \mathrm{~s}+1.419 \mathrm{e} 014}$ \\
7 & $\frac{1.163 \mathrm{e} 012 \mathrm{~s}+1.069 \mathrm{e} 016}{\mathrm{~s}^{2}+8.447 \mathrm{e} 009 \mathrm{~s}+2.658 \mathrm{e} 015}$ \\
8 & $\frac{7.328 \mathrm{e} 011 \mathrm{~s}+1.863 \mathrm{e} 016}{\mathrm{~s}^{2}+1.148 \mathrm{e} 010 \mathrm{~s}+4.369 \mathrm{e} 015}$ \\
9 & $\frac{3.002 \mathrm{e} 011 \mathrm{~s}+4.956 \mathrm{e} 015}{\mathrm{~s}^{2}+4.019 \mathrm{e} 009 \mathrm{~s}+1.076 \mathrm{e} 014}$ \\
10 & $\frac{2.293 \mathrm{e} 011 \mathrm{~s}+4.855 \mathrm{e} 015}{\mathrm{~s}^{2}+3.764 \mathrm{e} 09 \mathrm{~s}+5.159 \mathrm{e} 013}$ \\
\hline
\end{tabular}

\section{ILlustrative EXAMPLE OF A PWA CONTROL DESIGN FOR A PFC RECTIFIER}

The purpose of this section is to show that the formulation for controller synthesis presented in this paper is applicable to PWM PFC rectifiers. A boost PFC rectifier, with the values tabulated in Table I, has been built and tested. The proposed control system has been evaluated by simulations and experiments.

\section{A. The Voltage Loop Controller Design}

The voltage loop regulator can be designed starting from the large signal model of a PFC rectifier. This loop cannot attempt to remove the capacitor voltage ripple that occurs at the second harmonic of ac line frequency [18]. Therefore, for the purpose of designing the low-bandwidth outer control loop, it is unnecessary to model the high-frequency behavior of the system. It is desirable to model only the low-frequency components which are excited by slow variations in the control, the load and the line voltage amplitude. The highfrequency switching harmonics are removed by averaging the waveforms over a switching cycle. Hence, in the first stage, the waveforms are averaged over a switching period to remove the switching harmonics, and then in the second stage, averaging is used again over one-half of the ac line period to remove the even harmonics of the ac line frequency. The resulting model is valid for frequencies sufficiently less than the ac line frequency. The equivalent circuit is time-invariant but nonlinear. The signals can be perturbed and linearized to construct a small-signal ac model that describes how slow variations in the control signal, the load and the input affect the rectifier output. A small-signal equivalent circuit is given in Fig. 5. 


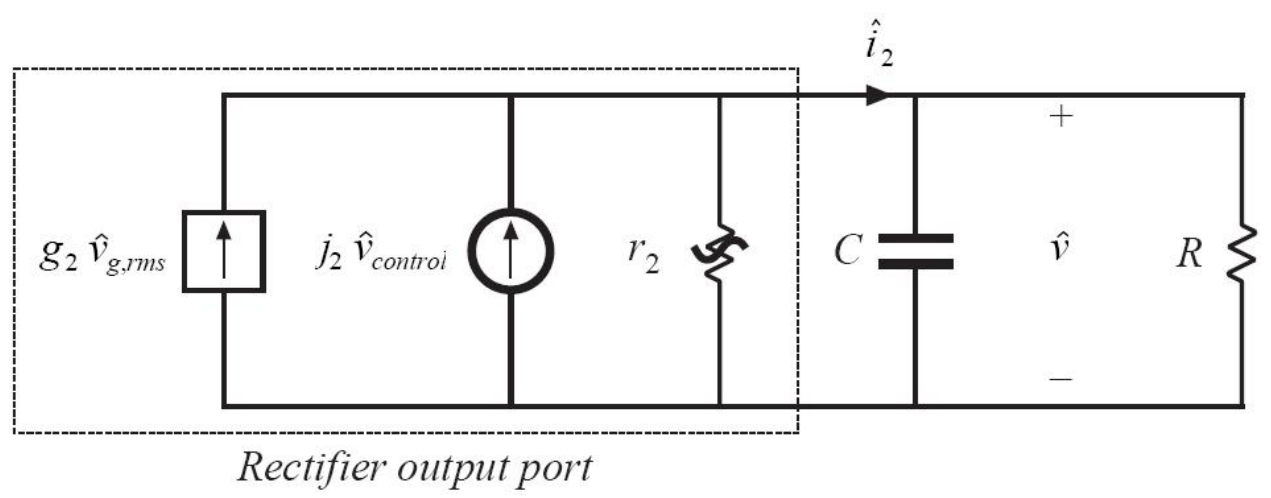

Fig. 5. The small signal equivalent circuit of the voltage loop [1].

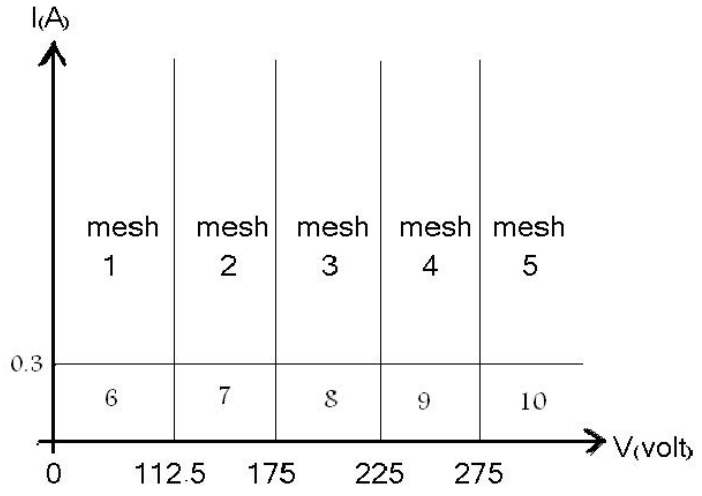

Fig. 6. State space partitioning.

Expressions for the parameters $\mathrm{g}_{2}, \mathrm{j}_{2}$, and $\mathrm{r}_{2}$, are given below [1]:

$$
\begin{gathered}
g_{2}=\frac{2}{R_{e}} \frac{V_{g}}{V} \\
r_{2}=\frac{V}{I_{2}} \\
j_{2}=\frac{V_{g, r m s}^{2}}{V R_{e}^{2}} \frac{d R_{e}}{d V_{\text {control }}} .
\end{gathered}
$$

The assumption that the inner current loop operates ideally is usually sufficient for designing a linear controller for the voltage control loop [1]. The following PI controller has been design for this purpose.

$$
G_{C V}(s)=\frac{\mathrm{s}+10}{\mathrm{~s}} .
$$

\section{B. The Current Loop Controller Design}

To arrive at a PWA approximation of the converter, the state space has been arbitrarily divided into 10 cells as illustrated in Fig. 6. Since in PFC rectifiers the current controller is designed to force the input current (state variable $x_{1}$ ) to be proportional to the input voltage $(u)$, partitioning for the input is not necessary.

Partitioning of the state space into smaller parts may lead to a better approximation. However, it also leads to a greater number of controllers and hence more complexity in controller synthesis.
Following the procedure in the previous section, a robust $\mathrm{H} H_{\infty}$ controller in sense of dynamic output feedback can be designed for each cell. To enforce continuity of the control inputs at the boundaries one can include actuator dynamics into the plant. The order of the system dynamics will then increase for each input [19]. Nevertheless, continuity of the control input is not a considerable issue for PWM converters. The step by step design procedure for PWA controllers can be summarized as follows:

1) Derive the averaged model of a PFC rectifier. For the boost rectifier the averaged model is described by (8) and (9).

2) Divide a part of the state space which is limited to the maximum and minimum operating current and the voltage into some rectangular cells.

3) Find the approximate piece-wise affine dynamics of the averaged model in each cell as in (12).

4) Find the corresponding augmented plant matrices in (13).

5) Solve the LMI optimization problem constructed by (17) through (19) using an appropriate solver. The output feedback controller for each cell (14) is obtained as a result of the optimization problem.

For the sample PFC rectifier the desired local controller for each cell is obtained as shown in Table II.

\section{Simulation Results}

In this section the steady state and dynamic performances of the proposed dynamically robust current control are studied by computer simulations. These simulations are carried out for the controller designed in the previous section.

Fig. 7 and Fig 8 show the steady state input current and output voltage for the proposed controller at the rated load. These simulated results show that the input current with the PWA controller has a low distortion. The DC output voltage after turning on under the rated load is shown in Fig. 9. The response of the voltage control loop is obviously fast with almost no overshoot.

\section{Experimental Results}

To verify the validity of the proposed controller, a boost PFC rectifier, with the same values and regulation circuits that were described in Table I, has been built and tested. The 


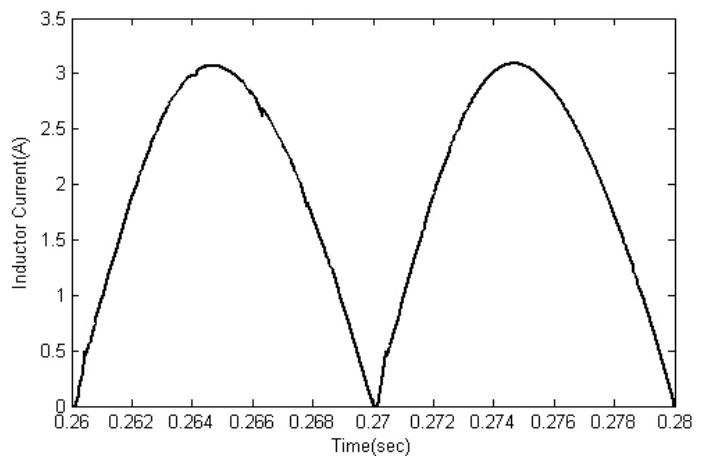

Fig. 7. Steady state input currents for the proposed control system.

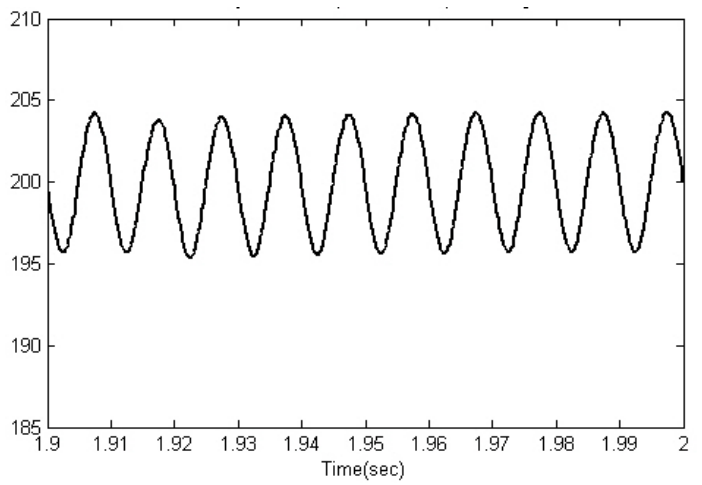

Fig. 8. Steady state output voltage for the proposed control system.

control system was designed around a TMS320F2812 Digital Signal Processor. The experimental setup together with a DSP evaluation board is shown in Fig. 10.

To arrive in discrete time controllers, the Tustin method is used. There are several methods for mapping from the splane to z-plane; such as; the forward and backward difference approximations. Tustin or bilinear approximation is one of the most popular methods which maps the entire left half of the s-plane into the unit circle in the z-plane [20].

Fig. 11 shows the waveforms of the input current and voltage into the rectifier circuit. The input voltage is intentionally kept at the lowest limit to emulate the worst case._It is evident that the average inductor current exactly follows the waveform of the reference current. The AC current contains negligible harmonic content. The THD is $4.8 \%$. The output voltage of the rectifier circuit is shown in Fig. 12. This voltage is precisely kept at the set point value.

\section{CONCLUSIONS}

In this paper, the subject of a PWA control design for PFC rectifier systems has been considered. A PWA model for a PFC rectifier circuit was developed. Then in terms of LMI, robust dynamic compensators for the approximated local models were designed to control the current loop. Computer simulations were performed to validate the proposed modeling and control approach. The results obtained show that the THD of the input current can be significantly improved with this control technique. The power factor rectifier can generate a close to unity power factor. An experimental prototype boost high power factor rectifier was built and tested to verify the theoretically predicted behaviors. The experimental results agree well with those of the computer simulation, which

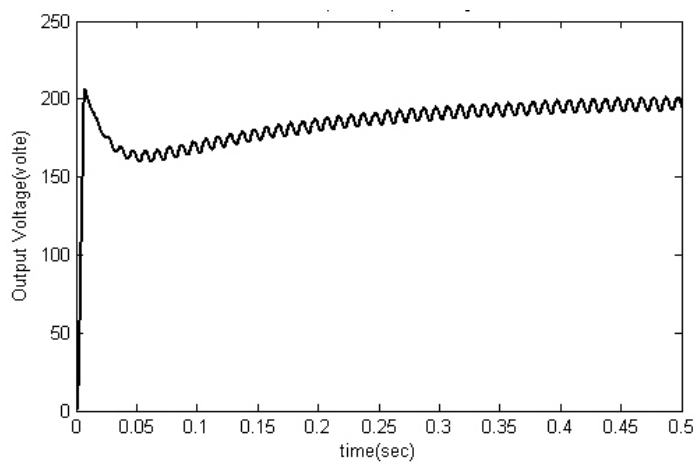

Fig. 9. Output voltage after turning on.

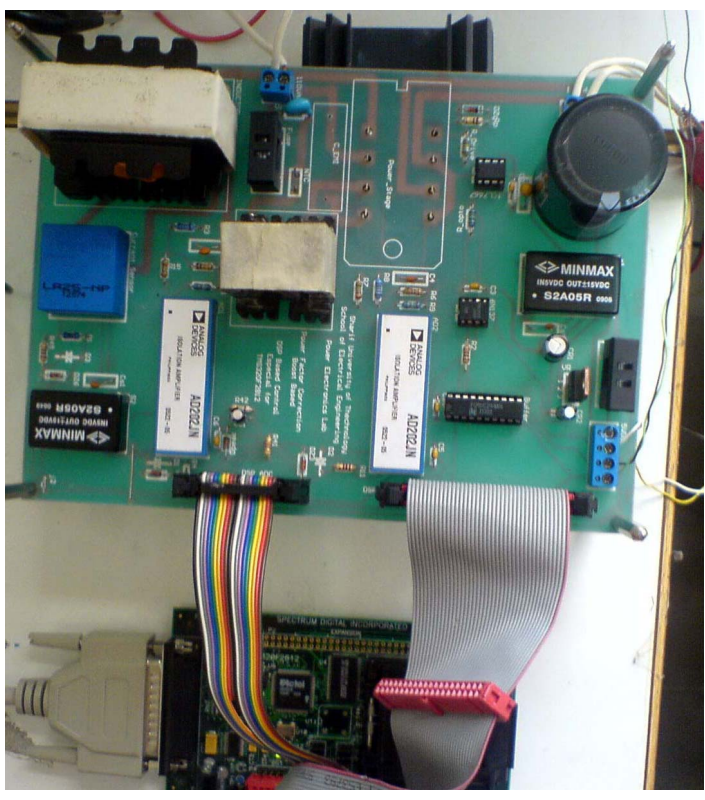

Fig. 10. The PFC rectifier with the DSP controller.

$50.00 \mathrm{~Hz}$ 02/07/09 08:52

\section{(1) $0.0 \%$ (4) $4.8 \%$}

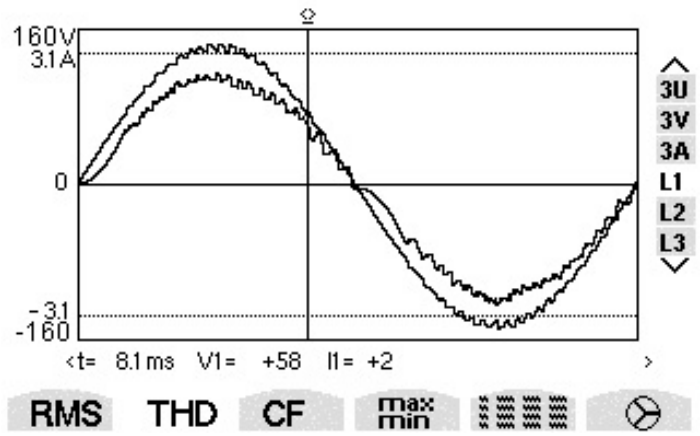

Fig. 11. Steady state input current (lower curve) and voltage of the experimental rectifier system with the proposed controller. 
$----\mathrm{Hz}$ 02/07/09 09:09 自? (1) $196.4 \mathrm{v}$

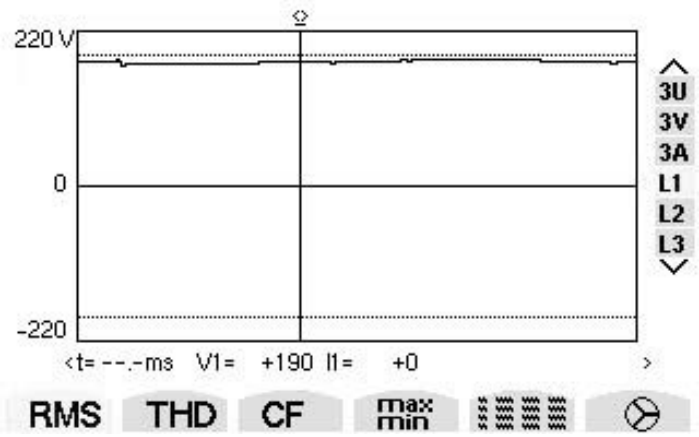

Fig. 12. Steady state output voltage of the experimental rectifier system with the proposed controller.

demonstrates the feasibility of the control technique. The results show low input current harmonics.

Although the proposed method was investigated on a Boost rectifier operating in CCM, this method can also be applied to other converters utilized in PFC rectifiers, such as Flyback, SEPIC and Cuk, where the small signal assumption of the inner current loop is violated. Furthermore the proposed method can be applied to converters operating in mixed conduction mode. When PFC rectifiers are operating under a light load, DCM will appear close to the crossover of the line voltage, causing the converter to switch between CCM and DCM. Since input current controllers are usually designed for operation in CCM, and the corresponding system transfer functions in CCM and DCM differ, the input current tracking will not be satisfactory. To avoid these problems, a large input inductor L can be exploited. Nevertheless, a converter with a low value input inductor $\mathrm{L}$ is desirable to reduce the weight and to allow for an easier design of the EMI-filter.

\section{REFERENCES}

[1] R. W. Erickson and D. Maksimovic, Fundamentals of Power Electronics, 2nd ed., Kluwer Academic Publishers, 2000.

[2] V. Vorperian, "Simplified analysis of PWM converters using the model of the PWM switch: Part I and II," IEEE Trans. Aerosp. Electron. Syst., Vol. AES-26, pp. 409-505, May 1990.

[3] A. Fernandez, J. Sebastian, P. Villegas, M. M. Hernando, and J. Garcia, "Dynamic limits of a power factor pre-regulator," in Proc. IEEE PESC'03, pp. 1697-1602, 2003.

[4] M. Alfayyoumi, A. H. Nayfeh, and D. Boroyevich, "Input filter interactions in DC-DC switching regulators," in Records of PESC'99, pp. 926-932, 1999.

[5] M. Chen, A. Mathew, and J. Sun, "Nonlinear current control of singlephase PFC converters," IEEE Trans. Power Electron., Vol. 22, No. 6, pp. 2187-2194, Nov. 2007.

[6] V. M. Rao, A. K. Jain, K. K. Reddy, and A. Behal, "Nonlinear control of a single phase unity power factor rectifier: design, analysis, and experimental results," IEEE Trans. Contr. Syst. Technol., Vol. 16, No. 6, pp.1301-1307, Nov. 2008.

[7] F.-Z. Chen and D. Maksimovic, "Digital control for improved efficiency and reduced harmonic distortion over wide load range in boost PFC rectifiers," Applied Power Electronics Conference and Exposition, 2009.

[8] A. Prodic, D. Maksimovic, and R. W. Erickson, "Dead-zone digital controllers for improved dynamic response of low harmonic rectifiers," IEEE Trans. Power Electron., Vol. 21, No. 1, pp. 173-181, Jan. 2006.
[9] A. Hassibi, S. P. Boyd, and J. P. How, "A class of lyapunov functionals for analyzing hybrid dynamical systems," in Proc. American Control Conference, Jun. 1999.

[10] F. Tahami and B. Molaei, "Piecewise affined system modeling and control of PWM converters," Journal of Circuits, Systems and Computers (JCSC), Vol. 16, No. 1, pp.113-128, Feb. 2007.

[11] L. Rodrigues and J. P. How. "Automated control design for a piecewise affine approximation of a class of nonlinear systems," in Proc. 2001 America Control Conference, 2001.

[12] J. C. Crébier, B. Revol, and J. Paul Ferrieux, "Boost-chopper-derived PFC rectifiers: Interest and reality," IEEE trans. Ind. Electron., Vol. 52, No. 1, pp. 36-45, Feb. 2005.

[13] A. Rantzer and M. Johansson, "Piecewise linear quadratic optimal control," IEEE Trans. Autom. Control, Vol. 45, No. 4, pp. 629-237, Apr. 2000.

[14] F. Tahami and H. M. Ahmadian, "Piecewise affine large signal modeling of PFC rectifiers," in Proc. 2007 IEEE International Symposium on Industrial Electronics (ISIE 2007), Jun. 2007.

[15] S. P. Boyd, L. El Ghaoui, E. Feron, and V. Balakrishnam, Linear Matrix Inequalities in System and Control Theory, Philadelphia, StatePA SIAM, 1994.

[16] C. Scherer, P. Gahinet, and M. Chilali, "Multiobjective output-feedback control via LMI optimization," IEEE Trans. on Autom. Control, Vol. 42, No. 7, pp 896-911, Jul. 1997.

[17] P. Gahinet, A. Nemirovski, A. Laub, and M. Chilali, The LMI Control Toolbox, The Mathworks Inc., 1995.

[18] R Ghosh and G. Narayanan, "A simple method to improve the dynamic response of single-phase PWM rectifiers," IEEE Trans. Ind. Electron. Vol. 55, No. 10, pp. 3627-3634, Oct. 2008.

[19] L. Rodrigues, A. Hassibi, and J. P. How, "Output feedback controller synthesis for piecewise-affine systems with multiple equilibria," in Proc. American Control Conference, 2000.

[20] C. H. Houpis and G. B. Lamont, Digital Control systems, New York: McGraw-Hill, 1985.

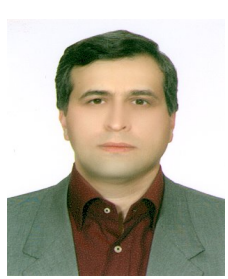

Farzad Tahami , was born in 1968, in Kashmar, Iran He received his B.S. in Electrical Engineering from the Ferdowsi University of Mashhad, Iran, in 1991, and his M.S. and Ph.D. in Electrical Engineering from the University of Tehran, Iran, in 1993 and 2003, respectively. From 1991 to 2004 he was with R\&D department of Jovain Electrical Machines Co. (JEMCO), Iran. In 2004 he joined the Sharif University of Technology, Tehran, Iran, where he is an Assistant Professor. His current research interest include electric motor drives, the modeling and control of power electronic converters, soft switching, resonant converters and vehicle system dynamics.

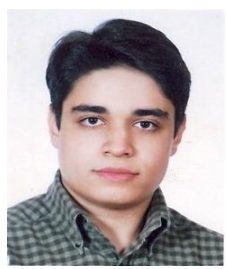

Shahab Poshtkouhi was born in Tehran, Iran, in 1987. $\mathrm{He}$ received his B.S. in Electrical Engineering from the Sharif University of Technology, Tehran, Iran, in 2009. He is currently pursuing his M.S. in Electrical Engineering at the University of Toronto, Canada. His current research interests include the digital control of PFC rectifiers and DC-DC converters, renewable energy and power electronics applications.

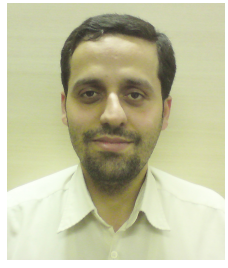

Hamed Molla Ahmadian was born in Mashhad, Iran, in 1982. He received his B.S. in Electrical Engineering from the Ferdowsi University of Mashhad, Iran, in 2005, his M.S. in Electrical Engineering from the Sharif University of Technology, Tehran, Iran, in 2008. He is currently pursuing his Ph.D. at the Ferdowsi University of Mashhad. He is engaged in research on the modeling and control of power electronic converters especially PFC rectifiers and DC-DC resonant converters. 